\title{
Ethical Values in Medicine
}

(Birdem Med J 2013; 3(1): 1-2)

Ethical consideration and trust between patients and physicians is a crucial aspect of medical practice. In recent years, Bangladesh has witnessed increasing discontent between physiciansand healthcare centers on the one hand and patients and public as a whole on the other hand. This is a reflection of the break of trust between providers and clients. Medical profession is not and should not be designed as a business. Prof. AK Azad Khan, Professor of Gastroenterology and President of Bangladesh Diabetic Somity, has rightly said in the oration delivered in memory of Late National Professor, Dr.Nurul Islam at the $24^{\text {th }}$ Annual Conference and Scientific Seminar of the Association of Physicians of Bangladesh, that physicians must persuade the Medical and Dental Council to be vigilant and perform their functions judiciously and more actively.

Medical profession is unique among all professions. It is a combination of science, art, skills and practice of ethical values. Medical practice has never been a commodity. This has given the profession its respectability.

In the beginning, medicine was mainly practiced as an art rather than a science, as physicians lacked the means to influence the course of diseases. Hippocrates prescribed a code of ethics for which he will always be remembered. But, unlike other philosophers like Socrates, Plato and
Aristotle, Socrates' thoughts lacked rationality regarding the cause and treatment of diseases. In contrast, in India, Charak, Shashruta and Vegabatta prescribed a code of ethics and at the same time were highly rational in their approach. It is essential to take note that ethics was the common thread linking these great thinkers. The professional associations should take lessons from this and ensure ethical medical practice.

As science progressed, physicians acquired effective agents for symptomatic relief, and thus began the practice of allopathy. With further advancements, especially during the last 50 years, there has been phenomenal progress about the understanding and the treatment of diseases. Some of these agents have powerful effects and if not used judiciously can cause great harm.

A legal limitation in Bangladesh is that Tort's law is not practiced in our country. This law deals with situations where a person's behavior has unfairly caused someone else to suffer loss or harm. The main remedy against tortuous loss is compensation for damages in monetary terms. This law can hence ensure compensation for cases involving unavoidable mistakes.

In conclusion physicians will only be considered successful when they practice their art not only skillfully but also ethically.

\section{MDR and XDR tuberculosis:}

Despite an encouraging progress in control, the global burden of TB remains enormous. Globally an estimated 12 million people were suffering from active TB in 2011, About 8.7 million new cases occurred in 2011, about $13 \%$ co-infected with HIV, deaths from TB was 1.4 million. Geographically, the burden of TB is highest in Asia and Africa. One important challenge in the fight against TB is multi-drug resistant TB (MDR-TB) and extensively drug resistant TB (XDR-TB).
MDR-TB is defined as tuberculosis that is resistant to at least Isoniazid and rifampicin. XDR-TB is defined as disease caused by Mycobacterium tuberculosis with resistance to a isoniazid and rifampicin, any fluoroquinolone, and at least one of three injectable second-line drugs (amikacin, capreomycin, or kanamycin). Globally $3.7 \%$ of new cases and $20 \%$ of previously treated cases are estimated to have MDRTB. According to WHO, in eastern Europe rate of MDR- 
TB are the highest, where MDR-TB makes 20 percent of all new cases.

As the tuberculosis chemotherapy era evolved, increasing cases of drug resistance continued to occur mainly as a result of inadequate regimens and nonadherence to therapy. Researchers initially suspected that these resistant organisms had reduced fitness and thus could be classified as being less virulent. This assumption was reversed in the 1990s with the rise in MDR tuberculosis. Substantial attention was focused upon New York City (NY, USA) where a virulent and transmissible strain had spread among immunocompromised populations. Awareness of tuberculosis drug resistance was refocused with a study presented in August, 2006, at the XVI International AIDS Conference in Toronto, Canada, which described an epidemic of XDR tuberculosis in a rural hospital in KwaZulu-Natal Province, South Africa.

To combat this situation a more focused and comprehensive strategy is needed. In Bangladesh the national tuberculosis control programme (NTP) introduced DOTS (Directly observed treatment short clourse) strategy in 1993 and gradually expanded. By mid 1998 it covered all upazillas. In 2002 DOTS started in Dhaka Metropolitan City and by 2006 the geographical Coverage was $100 \%$. In 2006; The STOP TB Strategy with special emphasis on NSN(new smear negative), EP(extra pulmonary), Child TB, MDR-TB and TB/HIV was taken. NTP objectives are detection of cases $>70 \%$ (Achieved in 2006), treat successfully $>85 \%$ (Achieved in 2003), reach MDG related targets of halving TB mortality and prevalence by 2015; So far NTP has established National TB Reference Lab (NTRL) at NIDCH, Dhaka.Regional TB Reference Lab (RTRL) at Rajshahi, Chittagong .One more is to be established soon in Khulna.

Number of Enrolled MDR-TB patients is1098 (up to December 2012). Treatment success rate up to 2009 cohort $=64 \%$

Country's 1st nationwide drug resistance survey conducted shows MDR 1.4\% \& $28.5 \%$ among new and previously treated TB cases respectively.

\section{References:}

1. Fact sheet: World TB day, 2011; Bangladesh tuberculosis Control Programme. (www.stoptb.org/..infactsheets/ fast.facts.asp.) 